\title{
Haemophagocytic Syndrome Secondary to Cytomegalovirus Infection in an Infant
}

\author{
Paul S1, Guha $\mathbf{S}^{2}$, Basu $\mathbf{S}^{3}$, Mukherjee $\mathrm{DK}^{4}$, Banerjee $\mathbf{R}^{5}$
}

${ }^{1}$ Dr. Subhasish Paul, MBBS, MD, Post graduate trainee, ${ }^{2} \mathrm{Dr}$. Suparna Guha, MD, Associate professor, ${ }^{3} \mathrm{Dr}$. Sumita Basu, MBBS. MD, Associate professor, ${ }^{4} \mathrm{Dr}$. Dilip Kumar Mukherjee, MBBS, DCH, MD, PhD, Professor; ${ }^{5} \mathrm{Dr}$. Raja Banerjee, MBBS, MD, Registrar. All from the department of Paediatrics, Vivekananda Institute of Medical Sciences, Kolkata, India.

\section{Address for correspondence:}

Dr. Subhasish Paul

E-mail: dr.subho2010@gmail.com

\section{How to cite}

Paul S, Guha S, Basu S, Mukherjee DK, Banerjee R. Haemophagocytic Syndrome Secondary to Cytomegalovirus Infection in an Infant. J Nepal Paediatr Soc 2016;36(1):88-90.

doi: http://dx.doi.org/10.3126/jnps.v36i1.14077

This work is licensed under a Creative Commons Attribution 3.0 License.

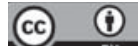

\begin{abstract}
Haemophagocytic syndrome caused by CMV infection in a one month old infant is rare. Cytomegalovirus can cause congenital or perinatal infection in an infant. We report a case in a baby who had secondary haemophagocytic syndrome. The baby had progressive pancytopenia, hepatosplenomegaly and diagnosed to be infected with CMV. Inspite of aggressive management the baby succumbed to death. CMV infection in a neonate may predispose to haemophagocytic syndrome.

Key words: Secondary haemophagocytic syndrome, Cytomegalo virus, Bone marrow aspiration.
\end{abstract}

\section{Introduction}

$\mathrm{H}^{2}$ aemophagocytic lymphohistiocytosis $(\mathrm{HLH})$ is an syndrome characterized by persistent fever, pancytopenia, liver dysfunction, hepatosplenomegaly, hyperferritinemia, hypofibrinogenemia, elevated serum lactate dehydrogenase, and hypertriglyceridemia ${ }^{1}$ with pathological finding of phagocytosis by macrophages of erythrocytes, leukocytes, platelets, and their precursors in bone marrow and other tissues $^{2}$. HLH comprises primary HLH (familial / genetic HLH) and secondary HLH. Secondary HLH could be due to infections, systemic onset rheumatic diseases and malignancies ${ }^{3}$.

We present a case of hemophagocytic syndrome secondary to cytomegalovirus infection in a one month old infant.

\section{The Case}

A one month five days old female infant delivered by LUCS for oligohydramnios at 33 weeks of gestation and weighing $1.2 \mathrm{~kg}$ at birth presented with progressive pallor and abdominal distension. Previous history revealed two spontaneous abortions and a history of NICU admission due to VLBW. Parents noted the distended abdomen after discharge on the eighth day of life. Antenatal history was uneventful, except for the mother being hypothyroid. On examination baby was alert but febrile. There was pallor and icterus but no cyanosis or oedema. Rash or hemorrhagic spots were noted all over body. Abdomen was tense, distended abdomen and umbilicus was everted. Liver was palpable $4.5 \mathrm{~cm}$ below right costal margin, which was soft along with a just palpable spleen. Other systemic examination was

J. Nepal Paediatr. Soc. 
non contributory. A provisional diagnosis of sepsis was made.

Table 1: Hematological examination revealed the following:

\begin{tabular}{|c|c|}
\hline Hemoglobin, & $4.8 \mathrm{~g} / \mathrm{dl}$ \\
\hline RBC indices & $\begin{array}{l}\text { Severe microcytosis and } \\
\text { hypochromia }\end{array}$ \\
\hline Peripheral blood smear & No abnormal cells. \\
\hline Platelets & 1.45lacs/cumm \\
\hline Total leucocyte count & $14,400 /$ cumm \\
\hline Reticulocyte count & $6.5 \%$ \\
\hline $\begin{array}{l}\text { Corrected reticulocyte } \\
\text { count }\end{array}$ & $2.7 \%$ \\
\hline CRP & 12mg/l (raised) \\
\hline Total bilirubin & $5.1 \mathrm{mg} / \mathrm{dl}$ \\
\hline Conjugated bilirubin & $2.8 \mathrm{mg} / \mathrm{dl}$ \\
\hline Albumin & $1.6 \mathrm{~g} / \mathrm{dl}$ \\
\hline ALP & $564 \mathrm{iu} / \mathrm{L}$ \\
\hline INR & 3.0 \\
\hline USG abdomen & $\begin{array}{l}\text { Hepatospleenomegaly and } \\
\text { free fluid in the abdomen. }\end{array}$ \\
\hline
\end{tabular}

Recurrent hypoglycaemic episodes were present after admission.

After PICU admission, three possibilities were kept in mind. Sepsis was the first diagnosis for which sepsis screen. In the background of severe IUGR and conjugated hyperbilirubinemia our second differential diagnosis was congenital TORCH infection, so TORCH screening was sent. In the light of recurrent hypoglycaemia the third differential diagnosis was galactosaemia. Patient was treated with IV fluids, Inj. Cefotaxim and then Inj. Meropenem. Packed RBC and fresh frozen plasma were also transfused as supportive treatment.

After transfusion $\mathrm{Hb}$ increased, the total count and platelet count also decreased Urine for non glucose reducing substance was positive serum ferritin was very high (3390 ng/ml). Hypoglycaemic episodes continued with no change in liver or spleen size. LFT and clotting profile remained deranged, and bilirubin levels kept rising. A diagnosis of congenital infection with Haemophagocytic lymphohistiocytosis was made.

Bone marrow aspiration cytology on D10 of admission showed histiocytes with evidence of haemophagocytosis. TORCH screening report showed CMV (IgM positive). A diagnosis of congenital CMV infection with secondary HLH was made. Breast milk was stopped and lactose and sucrose free formula feed was started for suspicion of galactosaemia, enzyme assay could not be done due to prior blood transfusion.
On Day 13 of admission, treatment was promptly started with oral Valgancyclovir $25 \mathrm{mg}$ twice daily for congenital CMV infection. Inspite of regular monitoring of absolute neutrophil count, platelets and serum creatinine level, patient had progressive worsening clinically with recurrent hypoglycaemia, jaundice, lethargy, abdominal distension. CMV PCR was also found to be positive in urine.

On day 15 of admission, $\mathrm{Hb}$ was $10.9 \mathrm{gm} / \mathrm{dl}$, total leucocyte count 5100/cumm, platelet count 1,50,000/ cumm, total bilirubin was $11.9 \mathrm{mg} / \mathrm{dl}$, conjugated bilirubin $4 \mathrm{mg} / \mathrm{dl}$,total protein $4.7 \mathrm{gm} / \mathrm{dl}$, albumin $2 \mathrm{gm} / \mathrm{dl}$, SGOT 118 U/L, ALP 837 U/L, INR was 3.94, APTT showed no coagulation. Fibrinogen was low. As there was further deterioration of the patient, we shifted the baby to PICU again. Intravenous immunoglobulin was administered on two successive days in order to treat HLH. On Day 19 baby started having epistaxis and haemoptysis. Inspite of receiving Valganciclovir and IVIG for $\mathrm{HLH}$, the patient developed DIC and expired on Day 19.

The presentation being early, the possibility of genetic/familial HLH could not be absolutely ruled out. Genetic analysis report of parents and baby to exclude primary or familial haemophagocytic lymphohistiocytosis was done and did not show any mutation in PRF 1 and UNC13 D coding exons.

\section{Discussion}

Haemophagocytic lymphohistiocytosis $(\mathrm{HLH})$ is a rare but potentially fatal disease of normal but overactive histiocytes and lymphocytes that commonly appears in infancy, although it has been seen in all age groups. Fever, hepatosplenomegaly, pancytopenia, lymphadenopathy, and rash often comprise the initial presentation. Cutaneous involvement occurs in as many as $65 \%$ of patients ${ }^{4}$. Primary HLH (ie, familial erythrophagocytic lymphohistiocytosis [FEL]), an inherited form of haemophagocytic lymphohistiocytosis syndrome, is a heterogeneous autosomal recessive disorder found to be more prevalent with parental consanguinity. Secondary haemophagocytic lymphohistiocytosis (ie, acquired haemophagocytic lymphohistiocytosis) occurs after strong immunologic activation, such as that which can occur with systemic infection, immunodeficiency, or underlying malignancy. Both forms are characterized by the overwhelming activation of normal $T$ lymphocytes and macrophages, leading to clinical and hematologic alterations and death in the absence of treatment ${ }^{5}$. Cytokine Storm Syndromes (CSS) are a group of disorders representing a variety of inflammatory etiologies of overwhelming systemic inflammation, hemodynamic instability, multiple organ dysfunction, 
and potentially death. The haemophagocytic syndromes haemophagocytic lymphohistiocytosis (HLH) and macrophage activation syndrome (MAS) represent two clinically similar CSS with an unknown degree of aetiopathologic overlap ${ }^{6}$. Familial $\mathrm{HLH}$, by definition, occurs in individuals with little to no ability of their Natural Killer (NK) cells and cytotoxic T-cells to kill targeted cells. Similar to SCID patients, children with $\mathrm{fHLH}$ will eventually succumb to their illness without eventual allogeneic bone marrow transplantation ${ }^{7}$.

According to the Proposed HLH criteria 2009, the following are the diagnostic criteria for HLH-

1. Molecular diagnosis of haemophagocytic lymphohistiocytosis (HLH) or X-linked lymphoproliferative syndrome (XLP).

2. Or at least 3 of 4 :
a. Fever
b. Splenomegaly
c. Cytopenias (minimum 2 cell lines reduced)
d. Hepatitis

3. And at least 1 of 4 :
a. Hemophagocytosis
b. $\uparrow$ Ferritin
c. $\uparrow$ sIL2Ra (age based)
d. Absent or very decreased NK function

4. Other results supportive of HLH diagnosis:
a. Hypertriglyceridemia
b. Hypofibrinogenemia
c. Hyponatremia

In our case there was hepatosplenomegaly, bicytopenia due to anaemia and thrombocytopenia, haemophagocytosis in bone marrow aspiration cytology,

\section{References}

1. Mashuku S, Hibi S, Todo S. Hemophagocytic lymphohistiocytosis in infancy and childhood. J Pediatr 1997; 130:352-7.

2. Favara BE. Hemophagocytic lymphohistiocytosis: a hemophagocytic syndrome. Semin Diagn 1992;9:6374.

3. Wong K, Chan JK. Reactive hemophagocytic syndrome- a clinicopathologic study of 40 patients in an oriental population. Am J Med 1992;93:177-80.

4. Morrell DS, Pepping MA, Scott JP. Cutaneous manifestations of hemophagocytic lymphohistiocytosis. Arch Dermatol 2002;138(9):1208-12.

5. Feldmann J, Le Deist F, Ouachee-Chardin M. Functional consequences of perforin gene mutations in 22 patients with familial haemophagocytic lymphohistiocytosis. Br J Haematol 2002;117(4):96572. hyperferritinaemia and hypofibrogenaemia. Patient also showed presence of CMV infection in serological investigation. As the patient came to us at the age of one month, whether it is a congenital or perinatally acquired CMV infection could not be determined. Mothers CMV infection status was also not done.

Cytomegalovirus (CMV) is the most common congenital infection in humans. It is also an established cause of $\mathrm{HLH}$ in infants or immunosuppressed population. Congenital CMV infection can follow either a primary or recurrent maternal infection, but the likelihood of foetal infection and the risk of associated damage is higher after a primary infection. As the infant presented at one month of age a possibility of primary $\mathrm{HLH}$, triggered by CMV infection was kept in mind. Less than $5 \%$ patients have severe inclusion disease. Jaundice, petechiae, and hepatosplenomegaly are the most frequently noted clinical triad in symptomatic infants ${ }^{8}$. Though there was non glucose reducing sugar present in urine, the diagnostic enzyme assay could not be done, as blood transfusion was done, and the patient expired within few days.

One similar case of hemophagocytic syndrome secondary to CMV infection was documented by Gupta A, Sen $R$ et al in journal of cytology, $2011^{9}$. Another case of HLH secondary to galactosemia in a three days old baby is also reported in $2012^{10}$.

\section{Conclusion}

In CMV infection secondary HLH may occur in infants, which may prove fatal even after treatment with valganciclovir. Whether galactosemia may cause secondary $\mathrm{HLH}$ that remains to be scrutinised.

6. Canna SW, Behrens EM. Making sense of the cytokine storm: a conceptual framework for understanding,diagnosing and treating hemophagocytic syndromes. Pediatr Clin North Am 2012;59(2):329-344. doi: 10.1016/j.pcl. 2012.03.002.

7. Henter JI, Horne A, Arico M, et al. HLH-2004: Diagnostic and therapeutic guidelines for hemophagocytic lymphohistiocytosis. Pediatr Blood Cancer 2007;48(2):124-131.

8. Leung KC, Sauve RS, Davies HD. Congenital cytomegalovirus infection. J Natl Med Assoc 2003;95(3): 213-218.

9. Gupta A, Sen R, Batra C,et al. J Cytol 2011;28(1):3638. doi:10.4103/0970-9371.76949

10. Kundak AA, Zenciroglu A, Yarali $\mathrm{N}$, et al. Turk J Haematol. 2012;29(4):401-404. doi: 10.5505/ tjh.2012.65148 